\title{
RESEARCH HIGHLIGHT AMPK hierarchy: a matter of space and time
}

\author{
David Carling (1D) \\ Cell Research (2019) 29:425-426; https://doi.org/10.1038/s41422-019-0171-6
}

\begin{abstract}
AMP-activated protein kinase (AMPK) is a key sensor of energy balance in eukaryotic cells, responding to low energy status by switching off anabolic pathways and upregulating catabolic processes. Zong and colleagues now show that different intensities of stimulation trigger activation of specific subcelIular pools of AMPK, resulting in phosphorylation of different downstream targets.
\end{abstract}

AMP-activated protein kinase (AMPK) is the downstream component of a protein kinase cascade that plays a central role in regulating energy homeostasis. Over the last few decades our appreciation of the regulation of AMPK and its function in coordinating multiple aspects of metabolism has expanded enormously. This increase in knowledge has led to significant interest in AMPK as a potential target for therapeutic intervention in a wide range of metabolic diseases, including obesity, type 2 diabetes, and cancer. ${ }^{1}$ However, a number of key issues concerning fundamental aspects of AMPK biology remain poorly understood. AMPK is a heterotrimeric complex consisting of three subunits: a catalytic subunit ( $\alpha$ ) and two regulatory subunits ( $\beta$ and $\gamma$ ). Isoforms of all three subunits exist in mammalian cells, but the physiological relevance of the different isoforms is only partially appreciated. AMPK is activated by phosphorylation of a threonine residue within the a subunit (Thr172) and this is catalyzed by either liver kinase b1 (LKB1) or $\mathrm{Ca}^{2+} /$ calmodulin-dependent protein kinase kinase 2 (CAMKK2). ${ }^{2}$ In contrast to CAMKK2, which is activated in response to increased intracellular $\mathrm{Ca}^{2+}$, LKB1 appears to be constitutively active. One mechanism by which AMPK phosphorylation by LKB1 can be increased is by slowing the rate of dephosphorylation of phosphorylated Thr172 (pThr172). A number of studies have shown that binding of AMP or ADP to the $\gamma$ subunit protects against dephosphorylation of pThr172 by protein phosphatases. ${ }^{3}$ In addition, AMP, but not ADP, has also been reported to increase the rate of phosphorylation of Thr172 by LKB1. This effect was shown to require interaction between LKB1 and axin inside the cell. ${ }^{4}$ Following this discovery, it was also shown that axin in complex with LKB1 translocates to the lysosomal surface via docking onto the Ragulator-v-ATPase complex. ${ }^{5}$ Remarkably, the axin-dependent lysosomsl pathway for AMPK activation turned out to be the mechanism for sensing the intracellular levels of glucose/fructose-1,6-bisphosphate (FBP) in an AMP-independent manner. ${ }^{6}$ This study provided a new paradigm for AMPK regulation, revealing that there are at least three pathways leading to AMPK activation: adenine nucleotide-dependent, $\mathrm{Ca}^{2+}$-dependent, and FBP-dependent.

In a recent study published in Cell Research by Zong et al. ${ }^{7}$ the authors build on their previous work and examine the biological relevance of the adenine nucleotide- and FBP-dependent activation pathways. They found that a reduction in intracellular FBP levels, but without an increase in AMP, results in specific activation of lysosomal-associated AMPK. Under these conditions, increased phosphorylation of a restricted subset of known AMPK substrates is observed. To further investigate this finding, phosphorylation of acetyl-CoA carboxylase (ACC) was monitored. There are two isoforms of ACC in mammalian cells. ACC1 is predominantly expressed in lipogenic tissues (e.g., liver, adipose), whereas ACC2 is predominantly expressed in tissues requiring high rates of fatty acid oxidation (e.g., skeletal muscle). In contrast to ACC1, which is a cytosolic protein, ACC2 is associated with mitochondria. ${ }^{8}$ Activation of lysosomal AMPK (following a reduction in FBP) led to increased phosphorylation of $A C C 1$, but not $A C C 2$, indicating a clear preference in downstream targeting. If cells were treated under conditions that caused both a reduction in FBP and a modest increase in AMP, activation of AMPK in both the lysosomal and cytosolic fractions was detected. Crucially, this activation was dependent on axin, and resulted in increased phosphorylation of ACC1, but not ACC2. In contrast, under severe nutrient restriction conditions that caused a much greater increase in intracellular AMP, AMPK was activated in the lysosomal, cytosolic, and mitochondrial fractions. Under these conditions, activation was not dependent on axin, and increased phosphorylation of both ACC1 and ACC2 was observed.

Taken together, these findings have a number of important implications. First, the study suggests that there exist distinct subcellular pools of AMPK that can be differentially activated depending on the metabolic status of the cell, specifically, the intracellular levels of FBP and AMP (Fig. 1). Lysosomal-associated AMPK is most sensitive to activation requiring only a decrease in FBP. Cytosolic AMPK shows an intermediate response requiring a modest increase in AMP (30-60 $\mu \mathrm{M})$ and mitochondrial-associated AMPK has low sensitivity, requiring high levels of AMP $(>100 \mu \mathrm{M})$. Notably, nuclear-associated AMPK was not activated under any of the conditions used in the study. Interestingly, a previous study reported that AMPK activation in response to energy stress is confined to the cytosol, whereas activation in both the cytosol and nuclear fraction was observed in response to increased intracellular $\mathrm{Ca}^{2+9}$. The FBP/low AMP activation pathways require axin, whereas activation in response to high AMP is axin independent. An important point raised in the study is that both axin 1 and axin2 can mediate the FBP/low AMP activation of AMPK by LKB1, whereas previous studies had focused specifically on axin1. How a modest rise in AMP concentration increases association of AMPK with axin/LKB1 remains unclear. There are two exchangeable nucleotidebinding sites within the $\gamma$ subunit of AMPK. Several lines of evidence suggest that one of these sites binds AMP with high affinity (low $\mu \mathrm{M})$ and the other with much lower affinity (high $\mu \mathrm{M}) .{ }^{10}$ Based on current knowledge, the nucleotide binding properties of the two sites do not provide an obvious mechanistic explanation for the axin-dependency of AMPK activation at different AMP levels. Finally, the study makes the important observation that activation of 


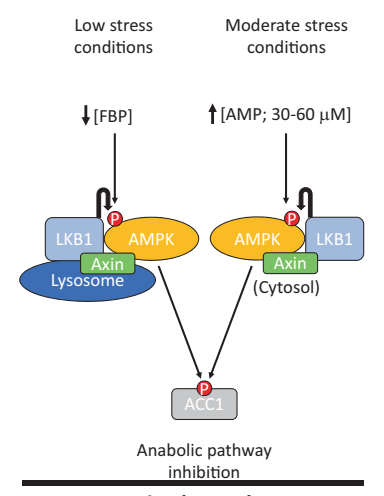

Axin-dependent

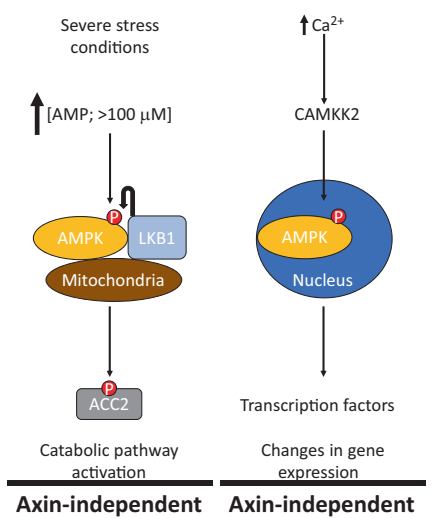

Fig. 1 Model for hierarchical activation of different subcellular pools of AMPK. Discrete pools of AMPK are activated by different intensities of stimulation: high-sensitivity (low stress) activates lysosomal-associated AMPK in response to a reduction in fructose 1,6 bisphosphate (FPB); medium-sensitivity (moderate stress) activates the cytosolic fraction of AMPK in response to a modest increase in AMP (30-60 $\mu \mathrm{M})$; low-sensitivity (severe stress) activates mitochondrial-associated AMPK in response to a marked increase in AMP $(>100 \mu \mathrm{M})$. Binding of LKB1 to axin is necessary for phosphorylation of AMPK on Thr172 by the high- and mediumsensitivity pathways, whereas the low-sensitivity pathway is axin independent. The different subcellular pools of AMPK phosphorylate distinct downstream targets, here exemplified by phosphorylation of either ACC1 (axin-dependent, lysosomal, and cytosolic pools) and ACC2 (axin-independent, mitochondrial pool). A potential implication of these findings is that the immediate effect of AMPK activation to low-stress conditions is inhibition of anabolic pathways (e.g., lipogenesis). Activation of catabolic pathways (e.g., fatty acid oxidation) occurs only after more severe metabolic stress. AMPK activation in the nucleus was not detected even after severe metabolic stress, and this is consistent with a previous study, ${ }^{9}$ which reported that AMPK activation in the nucleus required elevation of intracellular $\mathrm{Ca}^{2+}$. As shown here, it is possible that activation of AMPK in the nucleus is downstream of CAMKK2 signaling, which would likely be independent of axin, and could lead to changes in gene expression, mediated by phosphorylation of transcription factors distinct subcellular pools of AMPK can lead to differential phosphorylation of AMPK substrates. Increased phosphorylation of ACC2 is only detected in response to AMPK activation by high AMP (leading to activation of mitochondrial-associated AMPK). The authors speculate that this hierarchical substrate preference might indicate that the immediate role of AMPK activation is to switch off anabolic pathways, e.g., fatty acid synthesis, whereas activation of catabolic pathways, e.g., fatty acid oxidation, is a slower response. However, this is probably an over-simplification as previous studies using non-phosphorylatable mutants of ACC1 and ACC2 suggest that there is redundancy between ACC isoforms for both fatty acid synthesis and fatty acid oxidation. ${ }^{11}$ In addition, activation of fatty acid oxidation in vivo may occur in the absence of ACC2 phosphorylation, i.e., by the enhanced expression of CPT1 under starvation. As is often the case with major conceptual advances, the current findings raise a number of intriguing questions. For instance, do specific AMPK isoforms have an effect on subcellular localization and/or activation by different levels of AMP? In the context of therapeutic targeting, understanding these issues is likely to be an important consideration.

\section{ADDITIONAL INFORMATION}

Competing interests: The author declares that he has no conflict of interest.

\section{REFERENCES}

1. Steinberg, G. R. \& Carling, D. Nat. Rev. Drug. Discov. https://doi.org/10.1038/ s41573-019-0019-2 (2019).

2. Willows, R. et al. Biochem. J. 474, 3059-3073 (2017).

3. Carling, D., Mayer, F. V., Sanders, M. J. \& Gamblin, S. J. Nat. Chem. Biol. 7, 512-518 (2011).

4. Zhang, Y. L. et al. Cell Metab. 18, 546-555 (2013).

5. Zhang, C. S. et al. Cell Metab. 20, 526-540 (2014).

6. Zhang, C. S. et al. Nature 548, 112-116 (2017).

7. Zong, Y. et al. Cell Res. https://doi.org/10.1038/s41422-019-0163-6 (2019).

8. Abu-Elheiga, L. et al. Proc. Natl Acad. Sci. USA 97, 1444-1449 (2000).

9. Tsou, P., Zheng, B., Hsu, C. H., Sasaki, A. T. \& Cantley, L. C. Cell Metab. 13, 476-486 (2011).

10. Gu, X. et al. J. Biol. Chem. 292, 12653-12666 (2017).

11. Fullerton, M. D. et al. Nat. Med. 19, 1649-1654 (2013). 